\title{
Realizability of Graphs and Linkages
}

\author{
Marcus Schaefer \\ Department of Computer Science \\ DePaul University \\ 243 South Wabash \\ Chicago, Illinois 60604, USA \\ mschaefer@cdm.depaul.edu
}

\begin{abstract}
We show that deciding whether a graph with given edge lengths can be realized by a straight-line drawing has the same complexity as deciding the truth of sentences in the existential theory of the real numbers, ETR; we introduce the class $\exists \mathbb{R}$ that captures the computational complexity of ETR and many other problems. The graph realizability problem remains $\exists \mathbb{R}$-complete if all edges have unit length, which implies that recognizing unit distance graphs is $\exists \mathbb{R}$-complete. We also consider the problem for linkages: in a realization of a linkage vertices are allowed to overlap and lie on the interior of edges. Linkage realizability is $\exists \mathbb{R}$-complete and remains so if all edges have unit length. A linkage is called rigid if any slight perturbation of its vertices which does not break the linkage (i.e. keeps edge-lengths the same) is the result of a rigid motion of the plane. Testing whether a configuration is not rigid is $\exists \mathbb{R}$-complete.
\end{abstract}

\section{Introduction}

Many computational problems in geometry, graph drawing and other areas can be shown decidable using the (existential) theory of the real numbers; this includes the rectilinear crossing number, the Steinitz problem, and finding a Nash equilibrium; what is less often realized - with some exceptions - is that the existential theory of the reals captures the computational complexity of many of these problems precisely: deciding the truth of a sentence in the existential theory of the reals is polynomial-time equivalent to finding the rectilinear crossing number problem [3], solving the Steinitz problem [34, 4], finding a Nash Equilibrium [43], recognizing intersection graphs of convex sets and ellipses [42], recognizing unit disk graphs [32] and many 
other problems. ${ }^{1}$ In this paper we try to further substantiate this claim by showing that some well-known Euclidean realizability problems have the same complexity. One consequence of these results is that efficient algorithmic solutions to any of these problems are unlikely, since they would lead to efficient decision procedures for the existential theory of the real numbers, a problem that is NP-hard but not known (or expected) to be in NP.

\subsection{The Existential Theory of the Real Numbers}

The existential theory of the real numbers, ETR, is the set of true sentences of the form

$$
\left(\exists x_{1}, \ldots, x_{n}\right) \varphi\left(x_{1}, \ldots, x_{n}\right),
$$

where $\varphi$ is a quantifier-free $(\vee, \wedge, \neg)$-Boolean formula over the signature $(0,1,+, *,<, \leq,=)$ interpreted over the universe of real numbers. ${ }^{2}$

Tarski showed that ETR is decidable, but the running time of his decision procedure is not elementary (that is, bounded above by a tower of exponentials of fixed height). ${ }^{3}$ The existential theory of the reals is expressive enough to phrase many interesting problems in robotics and geometry, so research into more practical algorithms for deciding ETR has continued steadily since the 1970s when Collins discovered cylindrical algebraic decompositions that gave a double-exponential time algorithm for deciding ETR. Canny, motivated by problems in robot motion planning, showed that the problem is solvable in PSPACE [8]. This is still the best upper bound on ETR in terms of complexity theory, though Renegar sharpened Canny's result in terms of algebraic complexity [36, 37, 38]. For a detailed survey, see [33]; for experimental comparisons of running times, see [22].

As it turns out, ETR cannot only be used to solve algorithmic problems, it also captures the complexity of many such problems precisely. To make this statement precise, we use the notion of reducibility from computational complexity: we say a problem $A$ reduces to $B\left(A \leq_{\mathrm{m}} B\right)$ if there is a polynomial-time computable function $f$ so that $x \in A$ if and only if $f(x) \in B$ for all $x$; the function $f$ is known as the reduction. ${ }^{4}$ This notion

\footnotetext{
${ }^{1} \mathrm{~A}$ manuscript collecting many of these problems is in preparation [41].

${ }^{2}$ When writing formulas in the existential theory of the reals, we will freely use integers and rationals, since these can easily be eliminated without affecting the length of the formula substantially. We will also drop the symbol $*$.

${ }^{3}$ Tarski showed that the full theory of the reals is decidable by quantifier elimination.

${ }^{4}$ This reducibility is known as polynomial-time many-one reducibility; since we have no need for other reducibilities in this paper, we simplify to "reduces". We consider decision problems (requiring a "yes" or "no" answer) encoded as sets of binary strings;
} 
of reducibility is transitive, so we can use it to (partially) order problems by their complexity. Intuitively, $A$ reduces to $B$ if $A$ is at most as hard as $B$, since a solution to $B$ can be combined with the polynomial-time algorithm for $f$ to answer membership in $A .{ }^{5}$ We can now define $\exists \mathbb{R}$ to be the complexity class associated with ETR: a decision problem belongs to $\exists \mathbb{R}$ if it reduces to ETR; a decision problem is $\exists \mathbb{R}$-hard if every problem in $\exists \mathbb{R}$ reduces to it in polynomial time, it is $\exists \mathbb{R}$-complete if it belongs to $\exists \mathbb{R}$ and is $\exists \mathbb{R}$-hard. Analogously, we define notions based on $\forall \mathbb{R}$, the problems whose complement (in $\left.\{0,1\}^{*}\right)$ is in $\exists \mathbb{R}$. Note that $\mathbf{N P} \subseteq \exists \mathbb{R}$, since we can express satisfiability of a Boolean formula in $\exists \mathbb{R}$. For example, $(x \vee \bar{y} \vee z) \wedge(\bar{x} \vee y \vee z) \wedge(\bar{x} \vee \bar{y} \vee \bar{z})$ is equivalent to

$$
\begin{aligned}
& (\exists x, y, z)[x(x-1)=1 \wedge y(y-1)=1 \wedge z(z-1)=1 \\
& \wedge(x(1-y) z)+((1-x) y z)+((1-x)(1-y)(1-z))=0]
\end{aligned}
$$

We do not know, whether coNP $\subseteq \exists \mathbb{R}$. By Canny's result, $\exists \mathbb{R} \subseteq$ PSPACE and this is still the best known upper bound on $\exists \mathbb{R}[8]$.

\section{$1.2 \quad$ Stretchability and $\exists \mathbb{R}$-completeness}

To show that a geometric problem is $\exists \mathbb{R}$-complete we could try reducing from ETR, but that is typically hard. Moreover, it is unnecessary, since there is a prototypical geometric $\exists \mathbb{R}$-complete problem due to Mnëv: stretchability of pseudolines. A pseudoline is a simple curve which is $x$-monotone, that is, the curve crosses every vertical line exactly once. An arrangement of pseudolines is a finite collection of pseudolines so that any two pseudolines cross exactly once. (We allow multiple pseudolines to cross at the same point.) Two arrangements are equivalent if there is a homeomorphism of the plane turning one into the other. An arrangement of pseudolines is stretchable if it is equivalent to an arrangement of straight lines. We call the corresponding computational decision problem STRETCHABILITY.

Mnëv showed that ETR reduces to STRETCHABILITY. Shor [44] gives a much simpler proof of this result (also see [39]). In our terminology, we can express Mnëv's result as follows.

that is, $A, B \subseteq\{0,1\}^{*}$, and $f:\{0,1\}^{*} \rightarrow\{0,1\}^{*}$. For more background on encodings and basic definitions from computational complexity including complexity classes NP (nondeterministic polynomial time) and PSPACE (polynomial space), see any of the standard references, e.g. [35, 45]. We could have replaced polynomial time with logarithmic space in the definition of $\leq_{\mathrm{m}}$ but decided to use the more familiar notion.

${ }^{5}$ So " $A$ reduces to $B$ " does not mean that $B$ is easier than $A$, as the word "reduces" may incorrectly suggest. 
Theorem 1.1 (Mnëv [34]). STRETCHABILITY is $\exists \mathbb{R}$-complete.

Remark 1.2. Mnëv showed a much stronger result, his universality theorem, about the realizability of semi-algebraic sets through point-set configurations, of which Theorem 1.1 is a consequence. We will return to universality briefly in Section 2.3 when dealing with issues of precision.

Just because an arrangement is stretchable, it need not be easy to realize; indeed, Mnëv's universality theorem implies that there are stretchable arrangements of pseudolines so that every straight-line realization contains a non-rational line (that is, a line not containing a rational point; an earlier example of this is due to Perles according to Richter-Gebert and Ziegler [18, p.144]). It is known, however, that realizations cannot be arbitrarily complex. This is based on a result by Grigor'ev and Vorobjov [20] on semialgebraic sets. The total degree of a (multivariate) polynomial $f: \mathbb{R}^{n} \rightarrow \mathbb{R}$ is the maximum over the sum of variable exponents in each monomial term.

Theorem 1.3 (Grigor'ev, Vorobjov [20, Lemma 9]). If $f_{1}, \ldots, f_{k}: \mathbb{R}^{n} \rightarrow \mathbb{R}$ are polynomials each of total degree at most d and coefficients of bit-length at most $L$; then every connected component of $\left\{x \in \mathbb{R}^{n}: f_{1}(x) \geq 0, \ldots, f_{k}(x) \geq\right.$ $0\}$ contains a point of distance less than $2^{L d^{c n}}$ from the origin, for some absolute constant $c>0$.

The theorem (with proof) can also be found in [2, Theorem 13.15]. ${ }^{6}$ Based on this theorem, Goodman, Pollack, and Sturmfels [19] proved the following result. (They phrase the result for point configurations, which are dual to arrangements.)

Lemma 1.4 (Goodman, Pollack, Sturmfels [19]). A stretchable arrangement of $n$ pseudolines can be realized by $n$ straight lines so that all intersection points lie in the unit disk and so that the distance between any two intersection points and the distance of any intersection point and a line not containing that point is at least $1 / 2^{2^{c n}}$ for some fixed $c>0$.

Goodman, Pollack, and Sturmfels also showed the complementary result that some arrangements do require a precision of order $1 / 2^{2^{c n}}$ :

Lemma 1.5 (Goodman, Pollack, Sturmfels [19]). There are stretchable arrangements of $n$ pseudolines so that any straight-line realization of the arrangement for which all intersection points lie in the unit disk contains two intersections points within a distance less than $1 / 2^{2^{c n}}$ of each other for some fixed $c>0$.

\footnotetext{
${ }^{6}$ The statement in [2] contains a typo in the radius of the ball.
} 


\section{Realizability of Graphs}

Given a graph $G=(V, E)$ and a length $\ell(e) \in \mathbb{R}_{>0}$ for each $e \in E$, is there a straight-line drawing of the graph in the plane (not necessarily crossing-free) where each edge has its prescribed length? If so, we say that the graph is realizable in $\mathbb{R}^{2}$. Realizability depends on the notion of drawing we use; in the standard definition of a drawing, different vertices cannot coincide in the drawing and a vertex cannot lie on an edge unless it is an endpoint of that edge. ${ }^{7}$ If we do allow vertices to coincide and lie on edges, we enter the realm of linkages. For example, $K_{2,3}$ cannot be realized by a standard straight-line drawing in the plane if all edges have unit length, but it can be realized as a linkage with vertices overlapping. ${ }^{8}$ This section will center on graph realizability while Section 3 will discuss linkage realizability.

\subsection{Graph Realizability}

Theorem 2.1. Deciding whether a graph with given lengths is realizable is $\exists \mathbb{R}$-complete even if all edges have unit length.

Remark 2.2. We are not aware of any hardness results on the graph (as opposed to the linkage) realizability problem, though David Eppstein writes that he expects that "the Eades-Whitesides logic engine technique can be used to show that it's NP-hard to test whether a graph is a unit distance graph" [14]. There are results on plane realizations, which we survey in Section 2.4. The special case of the complete graph turns out to be efficiently solvable: Lemke, Skiena, and Smith sketch an algorithm that shows how to determine realizability of the complete graph and compute the coordinates of the points [29]. The history of complexity results on linkage realizability is discussed in Remark 3.2.

In the proof of Theorem 2.1 we make use of the Peaucellier linkage, a beautiful device transforming circular motion into linear motion. ${ }^{9}$ Figure 1

\footnotetext{
${ }^{7}$ For a discussion of graph drawing assumption, see [48].

${ }^{8}$ Our distinction between realizability of graphs and linkages is not universal, but not unusual; for linkages, [10] is a good reference; for graph realizability, definitions and terminology vary, e.g. realizable graphs are sometimes called Euclidean graphs. Often the definitions allow that vertices lie on edges of which they are not endpoints, though that may in some cases be due to oversight; as we will see, from a computational point of view, this distinction does not matter.

${ }^{9}$ There are many applets implementing Peaucellier's linkage available on the web to play with [30]. James Joseph Sylvester writes about Lord Kelvin that he "nursed it as if it had been his own child, and when a motion was made to relieve him of it, replied
} 
shows the linkage; we require $|a b|=|b c|,|a d|=|a f|$ and $|c d|=\mid$ de $|=| e f \mid=$ $|f c|$. If we keep points $a$ and $b$ fixed, and move $c$ (on a circular arc with center $b$ ), then $e$ moves on a straight line. In other words, the locus of $e$ is a straight line segment $[21]$.

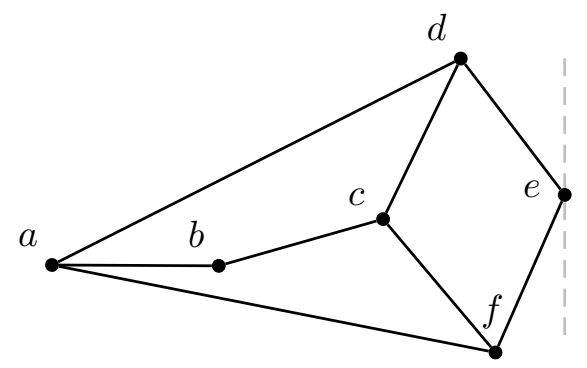

Figure 1: A Peaucellier Linkage

Figure 2 shows how to construct this Peaucellier linkage as a realizable graph with edges of unit length; we call this gadget $P_{a, b}(e)$ (edges $a d$ and af have been replaced by two rigid parts with unnamed vertices).

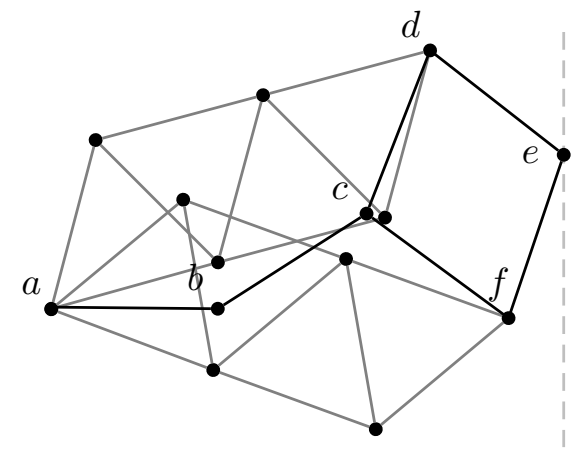

Figure 2: A Peaucellier Linkage with edges of unit length

Note that in the drawing all vertices have distinct positions and no vertex lies on an edge without being an endpoint of that edge. By continuity this remains true if we move $e$ slightly from its initial position. In general, we will need to place the Peaucellier gadget into partially completed drawings, and at that point we need to keep ensuring the basic graph drawing conventions.

"No! I have not had nearly enough of it - it is the most beautiful thing I have ever seen in my life." [47]. There have been other devices, both earlier and later, achieving the same effect, see [26] for an early history. 
We call a drawing nice if $(i)$ no vertex lies on a line of which it is not the endpoint, $(i i)$ no more than two edges cross in a point, (iii) two vertices that are not adjacent have a distance different from 1 .

With the Peaucellier gadget, we can build a "colinearity" gadget that ensures that three points lie on a line.

Lemma 2.3. There is a gadget $C\left(u_{1}, u_{2}, u_{3}\right)$ with edges of unit length so that in any realization of $C\left(u_{1}, u_{2}, u_{3}\right)$ the points $u_{1}, u_{2}$ and $u_{3}$ lie on a line. On the other hand, if three distinct points $u_{1}, u_{2}$ and $u_{3}$ lie on a line segment of length at most $\delta>0$, for some fixed $\delta$, then $C\left(u_{1}, u_{2}, u_{3}\right)$ is realizable. Moreover, $C\left(u_{1}, u_{2}, u_{3}\right)$ can be added to an existing nice drawing with three such vertices $u_{1}, u_{2}, u_{3}$ so that the resulting drawing remains nice.

Proof. We will use Peaucellier's linkage to guarantee colinearity: given three vertices $\left(u_{i}\right)_{i \in[3]}$ create three copies $\left(P_{i}\right)_{i \in[3]}$ of $P$, identify $a:=a_{1}=a_{2}=a_{3}$ and $b:=b_{1}=b_{2}=b_{3}$, and $u_{i}$ with $e_{i}, i \in[3]$ to obtain $C_{a, b}\left(u_{1}, u_{2}, u_{3}\right)$. Realizability of $C_{a, b}\left(u_{1}, u_{2}, u_{3}\right)$ guarantees that $u_{1}, u_{2}$, and $u_{3}$ are colinear by the properties of the Peaucellier gadget.

For the reverse direction, assume we start with a nice drawing containing three vertices $u_{1}, u_{2}, u_{3}$ lying on a sufficiently short line segment. Pick $a$ and $b$ so that $C_{a, b}\left(u_{1}, u_{2}, u_{3}\right)$ is realizable and nice (by itself) and remains so for small perturbations of $a b$. The resulting drawing may not be nice, since two non-adjacent vertices may have a distance of 1 , two distinct vertices overlap, or a vertex lies on an edge. Perturbing $a b$ slightly can destroy all three of these events, since it will perturb the locations of all the vertices in $C_{a, b}\left(u_{1}, u_{2}, u_{3}\right)$; the only pairs of vertices whose distance remains constant have an edge between them.

To encode STRETCHABILITY we need to express that a point $u_{2}$ lies between $u_{1}$ and $u_{3}$. For this we need another gadget $B\left(u_{1}, u_{2}, u_{3}\right)$.

Lemma 2.4. There is a gadget $B\left(u_{1}, u_{2}, u_{3}\right)$ with edges of unit length so that in any realization of $B\left(u_{1}, u_{2}, u_{3}\right)$ in which $u_{1}, u_{2}$, and $u_{3}$ lie on a line, $u_{2}$ lies between $u_{1}$ and $u_{3}$. On the other hand, if $u_{2}$ lies on the line segment $u_{1} u_{3}$ and $u_{1} u_{3}$ has length at most $\delta>0$, then $B\left(u_{1}, u_{2}, u_{3}\right)$ is realizable.

Proof. Consider the graph $B\left(u_{1}, u_{2}, u_{3}\right)$ shown in Figure 3. In any realization of that graph, $u u_{1}$ and $u u_{3}$ have distance 3 , while $u u_{2}$ has distance at most 3 , hence if all three points lie on the same line, then $u_{2}$ must lie between $u_{1}$ and $u_{2}$.

We can now complete the proof of Theorem 2.1. 


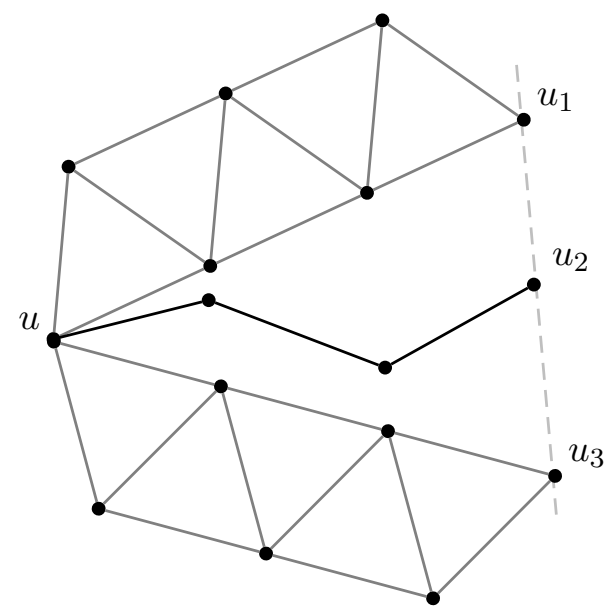

Figure 3: $B\left(u_{1}, u_{2}, u_{3}\right)$

Proof of Theorem 2.1. It is easy to see that the problem lies in $\exists \mathbb{R}$ (note that we do not have to calculate any square roots). We reduce from STRETCHABILITY: Suppose we are given a pseudoline arrangement $\mathcal{A}$. Create a vertex for every intersection point. For any three consecutive points $u_{1}, u_{2}, u_{3}$ along a pseudoline add the devices $C\left(u_{1}, u_{2}, u_{3}\right)$ and $B\left(u_{1}, u_{2}, u_{3}\right)$. Call the resulting graph $G_{\mathcal{A}}$ (all edges having unit length). If $G_{\mathcal{A}}$ is realizable, then a realization contains a set of line segments whose order of intersection correspond to $\mathcal{A}$. Since every two of these lines intersect (we included every intersection point in $G_{\mathcal{A}}$ ), we can extend these line segments to infinite straight lines without changing the order type, hence $\mathcal{A}$ is stretchable.

In the reverse direction, we have to show that if $\mathcal{A}$ is stretchable, then $G_{\mathcal{A}}$ is realizable. This would be entirely straightforward, if we did not have to ensure that the drawing is nice. Let us assume that $\mathcal{A}$ is stretchable. Then there is a realization by straight lines in which all intersections lie within the unit disk centered at the origin (using dilation). Let $D_{\delta}$ be the drawing containing all intersection points dilated by a factor of $\delta>0$, where $\delta$ is chosen sufficiently small for the $B$ and $C$ gadgets to work properly. Note that $D_{\delta}$ is a nice drawing, trivially so, since all points have distance less than 1 and there are no edges yet. To this drawing add the two rigid legs of each $B$ gadget, obtaining a drawing $D_{\delta}^{\prime}$. The resulting drawing may not necessarily be nice, since there could be two non-adjacent vertices which have a distance of 1 , two vertices that overlap, or a vertex lying on an edge. 
Let $p$ be a point on one of the gadgets $B$ ( $p$ does not have to be a vertex); we write $p(\delta)$, thinking of $p$ as depending on $\delta$. The coordinates of $p(\delta)$ are nearly polynomials in $\delta$, except that they may contain a term of the form $\sqrt{1-s \delta}$ for some fixed $s \in \mathbb{R}$ only depending on the placement of the $B$ gadget that $p$ belongs to in the initial drawing $D$. The square of the distance between two points (not necessarily belonging to the same $B$ ), or the square of the distance between a point and a line containing an edge (not necessarily belonging to the same gadget as the point) can similarly be expressed as a near-polynomial term in $\delta$ with at most 3 terms $\sqrt{1-s_{i} \delta}, i=1,2,3$. Hence, the condition that such a distance is 0 or 1 can be expressed using an equation which is nearly polynomial, except for at most three terms of the form $\sqrt{1-s_{i} \delta}$. However, three such terms can be removed (using symmetric polynomials), replacing the distance conditions with purely polynomial equations, which either have a finite number of solutions or are true for all $\delta$. As we let $\delta$ go to zero, the different $B$ gadgets converge to different locations (since the lines they are based on cannot be parallel: every pair of lines crosses), so it is not possible that two points belonging to different $B$ gadgets always have distance 0 or 1 , and similarly a point in one $B$ gadget cannot always have distance 0 from a line in another $B$ gadget. Finally, if two points within the same $B$ gadget always have distance 1 , they have an edge between them, and the distance between a point and a line in the same $B$ gadget changes with $\delta$, unless the point is an end-vertex of an edge of $B$. These observations imply that there are only a finite number of values of $\delta$ for which $D_{\delta}^{\prime}$ is not nice. Hence, we can pick an arbitrarily small $\delta>0$ for which the drawing $D_{\delta}^{\prime}$ is nice. Fix such a $\delta$.

We can now add the flexible middle leg of each $B$ gadget, so that the additional vertices maintain niceness (this is why the middle leg has two interior vertices: to be flexible enough to maintain niceness).

Finally, Lemma 2.3 allows us to add all the $C$ gadgets one by one, maintaining niceness of the drawing. This shows that $G_{\mathcal{A}}$ is realizable, even fulfilling the stronger condition that every pair of non-adjacent vertices has a distance different from 1 .

Corollary 2.5. The problem of graph realizability remains $\exists \mathbb{R}$-complete even if $(i)$ we do not require that vertices not lie on edges they are not endpoints of and (ii) non-adjacent vertices must have a distance different from 1.

Proof. This follows from the proof of Theorem 2.1: the proof never used assumption $(i)$ and when constructing the realization of $G_{\mathcal{A}}$, we ensured that non-adjacent vertices have distance different from 1 . 


\subsection{Euclidean Dimension and Unit Distance Graphs}

Let $E^{n}$ be the infinite graph on vertex set $\mathbb{R}^{n}$ so that $x y$ is an edge of $E^{n}$ if and only if $|x-y|=1$. Then Corollary 2.5 implies that recognizing subgraphs or induced subgraphs of $E^{2}$ is $\exists \mathbb{R}$-complete (independent of how we arbitrate the issue of vertices lying on edges). The Euclidean dimension of a graph $G$ is the smallest $n$ so that $G$ is a (induced) subgraph of $E^{n}$, a notion introduced by Erdős, Harary, and Tutte [16]. ${ }^{10}$

Corollary 2.6. Deciding whether a graph has Euclidean dimension 2 is $\exists \mathbb{R}$ complete (in both the induced and non-induced version). Hence, computing the Euclidean dimension of a graph is $\exists \mathbb{R}$-complete.

Subgraphs of $E^{2}$ are also known as unit distance graphs [9], strict unit distance graphs are induced subgraphs of $E^{2}$. The following is just a restatement of the preceding corollary, answering a question suggested by Eppstein [14].

Corollary 2.7. Recognizing (strict) unit distance graphs is $\exists \mathbb{R}$-complete.

Consequently, it is very unlikely that we will be able to recognize unit distance graphs efficiently [24].

Remark 2.8. We can think of unit distance graphs as graphs whose edges are labeled "=1"; this suggests looking at alternative label sets. For example, if edges can have labels " $<1$ " and "> 1 " instead of "= 1" we get unit disk graphs; McDiarmid and Müller [32] recently showed that SIMPLE STRETCHABILITY (in which no more than two pseudolines are allowed to intersect in a point) reduces to the recognition problem for unit disk graphs, so the unit disk graph problem is also $\exists \mathbb{R}$-complete, since SIMPLE STRETCHABILITY and STRETCHABILITY are polynomial-time equivalent [43].

\subsection{Issues of Precision}

The reduction from STRETCHABILITY to graph realizability in the proof of Theorem 2.1 is geometric in the following sense: each realization of the graph with length constraints constructed from the pseudo-line arrangement

\footnotetext{
${ }^{10}$ Erdös, Harary, and Tutte [16] defined the dimension of a graph using (non-induced) subgraphs of $E^{n}$. Later, Erdős and Simonovits [15] introduced Euclidean dimension under the name faithful dimension. The two notions differ: take a wheel $W_{6}$ with six spokes and remove one of the spokes. The resulting graph is realizable as a subgraph, but not as an induced subgraph of $E^{2}$. The name "Euclidean dimension" seems to be due to Maehara [31]. For details and more terminology and history, see [46, Section 13.2].
} 
encodes a straight-line realization of the arrangement. More precisely, the position of certain vertices of the graph encode the locations of intersection points of the straight-line realization. In other words, the intersection points of a realization can be obtained by projecting onto certain points of the graph. Lemma 1.5 now immediately implies that some graph realizations have exponentially low vertex resolution: the ratio between the maximum distance of any two vertices divided by the minimum distance between any two (distinct) vertices can be of order $2^{2^{c n}}$. Similarly, the reductions to Euclidean dimension and unit distance graphs are geometric. We state the result for unit distance graphs only.

Corollary 2.9. There are unit distance graphs on $n$ vertices so that any realization of the graph contains two distinct vertices at distance at most $1 / 2^{2^{c n}}$ for some fixed constant $c>0$.

Some of the traditional ETR results are actually universality theorem; for example, Mnëv [34] showed that any semi-algebraic set is stably equivalent to the realization space of a pseudoline arrangement. We do not want to define stable equivalence explicitly (see [39] for a detailed discussion), but roughly speaking it means that the two sets look very similar algebraically. Stable equivalence is not immediately useful to our purposes, since it does not imply any complexity bounds, but many of the universality theorems in the literature could be recast as polynomial-time many-one reductions. We also think it likely that many of our geometric reductions can be turned into universality theorems with some additional effort. We discuss universality theorems for linkages in Remark 3.3.

\subsection{Plane Realizations and Matchstick Graphs}

It is natural to ask what happens if we require the realizations of the graphs to be plane, that is, free of crossings. Strengthening earlier results by Whitesides [49] and Eades and Wormald [13], Cabello, Demaine, and Rote [7] showed that the plane realizability problem is (strongly) NP-hard even when restricted to 3-connected, infinitesimally rigid planar graphs with unit edge lengths. Plane realizable graphs with unit edge lengths - plane unit distance graphs - are known as matchstick graphs.

It remains open whether recognizing matchstick graphs, or solving the general plane realizability problem, is $\exists \mathbb{R}$-complete. If the graph is a triangulation, then Cabello, Demaine, and Rote [7] show that plane realizability can be decided in polynomial time in the real RAM model, and in $\mathbf{P}$, if the graph has bounded degree. Their proof can be modified to show that the 
problem lies in coRP without assuming bounded degree; a problem lies in coRP if there is a probabilistic algorithm which is correct if it says "no" and is correct with probability at least $1 / 2$ if it says "yes" [35, 45]. Running the algorithm repeatedly yields an algorithm with arbitrarily small error bound.

Lemma 2.10. If $G$ is a triangulation with prescribed lengths, then plane realizability can be tested in $\mathbf{c o R P}$, even if the edge lengths include square roots of rationals.

Our proof adapts an argument from Cabello, Demaine, and Rote [7].

Proof. By a result of Di Battista and Vismara [12] it is sufficient to verify the triangle inequality in each triangle, and ensure that the sum of angles at each interior vertex is $2 \pi$. Since the graph is a triangulation, we can easily determine its topological embedding (if there is a non-triangle face, it has to be the outer face, if all faces are triangles, we can try each of them as the outer face in polynomial time). Now pick an interior vertex $v$ and consider one of its incident triangles; the angle $\alpha$ formed by the triangle at $v$ fulfills the law of cosines: $\cos \alpha=\left(b^{2}+c^{2}-a^{2}\right) / 2 a b$, with standard notation for the triangle. Consequently, $\sin \alpha=\sqrt{1-\left(\left(b^{2}+c^{2}-a^{2}\right) / 2 a b\right)^{2}}$. Suppose the angles at $v$ are $\alpha_{i}, i \in[n]$, and let $A_{k}:=\sum_{i=1}^{k} \alpha_{i}$. We can then write recursive expressions for $\sin A_{k}$ and $\cos A_{k}$ using identities $\sin (\alpha+$ $\beta)=\sin \alpha \cos \beta+\cos \alpha \sin \beta$ and $\cos (\alpha+\beta)=\cos \alpha \cos \beta+\sin \alpha \sin \beta$. This expression can be viewed as a directed acyclic graph whose leaf nodes are the only nodes containing the square root operation (note that this remains true even if the input lengths are square roots of rationals). Blömer [5, Theorem 2.2] showed that deciding whether such an expression equals 0 lies in coRP. In particular, testing whether $\sin A_{n}=0$ and $\cos A_{n}=1$ is in coRP. We also need to ensure that all $A_{k}<2 \pi$, but it is sufficient to do this approximately, since the test of $\sin A_{n}=0$ and $\cos A_{n}=1$ guarantees that $A_{n}$ is a multiple of $2 \pi$, and the approximate test can be done in polynomial time.

If recognizing matchstick graphs were $\exists \mathbb{R}$-complete and the reduction were geometric, then, similarly to Corollary 2.9, we would need to be able to construct matchstick graphs in which vertices are forced to be exponentially close. This would be a first indication of potential $\exists \mathbb{R}$-hardness.

Question 2.11. Can one construct a matchstick graph on $n$ vertices so that in every plane realization of the graph there are two vertices of distance at most $1 / 2^{2^{c n}}$ for some fixed constant $c$ ? 


\section{$3 \quad$ Realizability of Linkages}

A linkage is a graph $G=(V, E)$ with a length $\ell(e)$ assigned to each edge $e \in E$; edges of a linkage are also called rods or bars; a configuration, or realization, of $G$ in $\mathbb{R}^{2}$ is a mapping of $V$ to $\mathbb{R}^{2}$ so that the distance between the endpoints of each edge $e$ equals $\ell(e)$. If there is a configuration of the linkage, we call the linkage realizable; our terminology is based on the exposition in [10]; a more detailed treatment can be found in [11]. We discuss two computational problems related to linkages: realizability, in Section 3.1 and rigidity, in Section 3.2.

\subsection{Linkage Realizability}

Theorem 3.1. Deciding whether a linkage is realizable is $\exists \mathbb{R}$-complete even if all edges have unit length.

The $\exists \mathbb{R}$-hardness of linkage realizability can also be obtained from universality results on linkages, see the discussion in Remark 3.3); the main new contribution in Theorem 3.1 is the restriction to unit lengths (and a simpler proof).

Remark 3.2. NP-hardness of linkage realizability was shown by Yemini [50] and Saxe [40]. Saxe showed that linkage realizability in $\mathbb{R}$ is NPcomplete even if distances are restricted to values 1,2 : let $\mathbf{w}:=\left\{w_{1}, \ldots, w_{n}\right\}$ be an instance of the partition problem and create the graph $C_{n}$ on edges $e_{1}, \ldots, e_{n}$ with $\ell\left(e_{i}\right)=w_{i}$. Then $C_{n}$ can be realized in $\mathbb{R}$ if and only if $\mathbf{w}$ is a positive instance of partition. Using gadgets with edges $a b, b c$ and $a c$, where $w(a b)=w(b c)=1$ and $w(a c)=2$ we can replace edges of arbitrary integer length with edges of lengths 1 and 2. Saxe extended this construction to show that linkage realizability in $\mathbb{R}^{k}$ for linkages with distances 1,2 is $\mathbf{N P}$-hard for all $k \geq 1$. He also discusses approximation results and results on how hard it is to decide whether there is a unique solution. Abbott [1] showed that deciding whether a linkage is rigid is hard; we discuss his result later in this section.

In the algebraic community, the linkage realizability problem is known as the Euclidean distance matrix completion problem (EDMCP); research in that community seems to concentrate on algebraic characterizations and using tools such as semi-definite programming to solve instances of the problem, see [28] for a survey.

Remark 3.3. Kempe claimed his universality theorem for linkages in 1876 . Roughly speaking, it shows how to trace any (compact) algebraic curve with 
a linkage, so linkages are universal for (compact) algebraic curves. ${ }^{11}$ This suggests a proof of $\exists \mathbb{R}$-hardness for linkage realizability: to test whether a polynomial $f$ can take on the value 0 extend the linkage tracing $f$ so that the vertex tracing $f$ is forced to lie on the line corresponding to $f=0$. Then $f=0$ has a solution if and only if the extended linkage is realizable. This approach can be made to work, however, there are some obstacles to overcome; first, Kempe's proof is incomplete (some of his gadgets have degenerate configurations). Jordan and Steiner [23] and Kapovich and Millson [25] gave the first correct and complete proofs of Kempe's universality theorem (and, in Kapovich and Millson's case even stronger results). The next obstacle is that these papers did not analyze the effectiveness of the constructions; Gao and Zhu [17] analyzed Kempe's proof and showed that in the plane $O\left(n^{4}\right)$ links are sufficient (also see Abbott [1, Section 1.5]), this still does not show that the lengths of the links can be calculated effectively (or can be assumed to be algebraic or rational numbers). The first detailed analysis of this aspect seems to be in Abbott's thesis [1] which we will discuss again below with respect to rigidity. Abbott's version of Kempe's universality theorem can be extended to a proof of $\exists \mathbb{R}$-hardness of linkage realizability as sketched above. However, the approach is quite intricate (after all, a much stronger result is obtained: universality); our proof below is more direct, and, moreover, it shows that unit lengths are sufficient to get $\exists \mathbb{R}$-hardness, while, as far as I know, there are no universality theorems for unit linkages.

The proof of Theorem 3.1 requires some modifications to the proof of Theorem 2.1; in some respects, the proof becomes easier, since we no longer have to ensure that vertices do not accidentally overlap with other vertices or edges in realizations. On the other hand, we need a new device that guarantees that vertices of linkages are mapped to distinct points of the plane.

In the construction we will use a small set of radii (the particular set of radii is rather arbitrary), so we first show that if we are given a linkage with multiple integer lengths, we can replace it with a unit length linkage without affecting realizability.

Lemma 3.4. Given a linkage $G$ with integer lengths, we can construct a linkage $G^{\prime}$ with unit lengths only, so that $G$ is realizable if and only if $G^{\prime}$ is realizable. The size of $G^{\prime}$ is polynomial in the size of $G$ and the integer lengths (in unary).

Proof. Let $M$ be the Moser spindle shown in Figure 4.

\footnotetext{
${ }^{11}$ See [25] or [11, Section 3.2.1] for detailed expositions.
} 


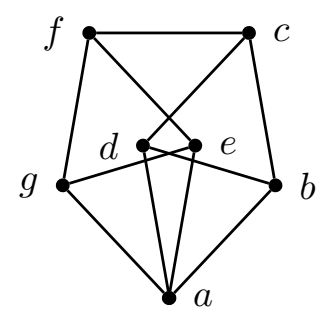

Figure 4: The Moser graph

The seven vertices of $M$ are distinct in any realization of $M$ in the plane: obviously the three vertices in each of the four triangles are distinct. Now the distance between $a$ and $c$ is either 0 or $\sqrt{3}$, and the same is true for $a$ and $f$. So it is not possible that one of $c$ or $f$ or both of them coincide with $a$, since $c$ and $f$ have a distance of 1 . Hence, both $c$ and $f$ are distinct from $a$, and this already forces all vertices of $M$ to be pairwise distinct. In particular, the diamond on $\{a, b, c, d\}$ does not collapse. We can thus chain several Moser graphs together by identifying them along triangles to create pairs of vertices at arbitrary integer distances. This allows us to replace an edge of length $n$ in $G$ using a gadget with less than $7(2 n-1)$ vertices.

Lemma 3.4 helps us resolve the problem of keeping vertices distinct: we cannot simply paste two triangles together along an edge, since the resulting diamond linkage can be realized by a single triangle, with two of the vertices (and two pairs of edges) coinciding. With the Moser graph we can avoid collapses of this type. For the proof of Theorem 3.1 we need devices that keep arbitrary pairs of vertices apart in a realization. This problem comes in two types: vertices like the two vertices in the diamond that are either identical or far apart, and vertices that could potentially be very close in a realization, but are not allowed to coincide. The second problem is more difficult, and we will show how to resolve it presently, the first problem only occurs in the construction of the Peaucellier linkage where we resolve it ad-hoc.

Lemma 3.5. We can create a linkage $P^{\prime}$ with $V\left(P^{\prime}\right)$ including $a$, $b$, and $e$ so that if $a$ and $b$ are fixed in the plane, then the locus of $e$ is a line segment of length at least 1.

Proof. We start with the Peaucellier linkage $P$ shown in Figure 2 assigning each edge a length of 2 . In a linkage realization of $P$ several pairs of points that need to be distinct for the gadget to work can collapse; this includes 
the diamonds connecting $a$ to $d$ and $a$ to $f$ as well as the pairs $d, f$ and $c, e$. Suppose $x$ and $y$ is one of these pairs; then we add a rather crude device to the linkage: add edges $x x^{\prime}, x^{\prime} y^{\prime}$ and $y^{\prime} y$ of lengths $w\left(x x^{\prime}\right)=1, w\left(x^{\prime} y^{\prime}\right)=3$, and $w\left(y^{\prime} y\right)=1$. In any realization, this forces $x y$ to have distance at least 1 (and $x$ and $y$ can have distance up to 5 which is sufficient for all realizations of $P$ ). The resulting linkage $P^{\prime}$ fulfills the statement of the lemma.

With the Peaucellier linkage we can now construct a linkage $L\left(u_{1}, u_{2}, u_{3}\right)$ that combines the functionality of the earlier colinearity and betweenness gadgets $B\left(u_{1}, u_{2}, u_{3}\right)$ and $C\left(u_{1}, u_{2}, u_{3}\right)$; as opposed to these earlier gadgets, the new device does not guarantee that $u_{2}$ is strictly between $u_{1}$ and $u_{3}: u_{2}$ could coincide with either. This is a problem we fix later.

Lemma 3.6. There is a linkage $L\left(u_{1}, u_{2}, u_{3}\right)$ with lengths in [4] so that the realizability of $L\left(u_{1}, u_{2}, u_{3}\right)$ implies that $u_{2}$ lies on the line segment $u_{1} u_{3}$ (including endpoints), while, in the reverse direction, if $u_{2}$ does lie on the line segment $u_{1} u_{3}$ and $u_{1} u_{3}$ has length at most 1 , then $L\left(u_{1}, u_{2}, u_{3}\right)$ is realizable.

Proof. Given three vertices $\left(u_{i}\right)_{i \in[3]}$ create three copies $\left(P_{i}^{\prime}\right)_{i \in[3]}$ of $P^{\prime}$ as constructed in Lemma 3.5, identify $a:=a_{1}=a_{2}=a_{3}$ and $b:=b_{1}=$ $b_{2}=b_{3}$, and $u_{i}$ with $e_{i}, i \in[3]$. Realizability of this part guarantees that $u_{1}, u_{2}, u_{3}$ are colinear. To ensure that $u_{2}$ lies between $u_{1}$ and $u_{3}$ we add two new vertices $g, h$ and edges $g h, h u_{2}, g u_{1}, g u_{3}$ with $w(g h)=w\left(h u_{2}\right)=$ 2 , and $w\left(g u_{1}\right)=w\left(g u_{3}\right)=4$. The resulting device is $L\left(u_{1}, u_{2}, u_{3}\right)$. If $L\left(u_{1}, u_{2}, u_{3}\right)$ is realizable, then $u_{2}$ lies on $u_{1} u_{3}$ (including endpoints), and if $u_{1} u_{3}$ has length at most 1 and $u_{2}$ lies on that segment, then $L\left(u_{1}, u_{2}, u_{3}\right)$ is realizable.

Finally, we need a gadget $D\left(u_{1}, u_{2}\right)$ that guarantees that $u_{1}$ and $u_{2}$ are distinct, where $u_{1}$ and $u_{2}$ are two intersection points. In general, such a gadget does not exist: if the loci of $u_{1}$ and $u_{2}$ can come arbitrarily close, then they must intersect, since both are compact sets. However, for realizations of stretchable arrangements, we have Lemma 1.4 which gives us a lower bound on how close intersection points need to get, and we can use that to build a device that simulates true "distinctness" well enough to work for our construction.

Lemma 3.7. There is a linkage $T(a, b)$ with lengths in [2] and $\{a, b, c\} \subset$ $T(a, b)$ so that if we assign $a$ and $b$ to any two points of distance less than 1 in the plane, then $a$ and $c$ have distance $|a-b|^{2}$ in any realization of $T(a, b)$.

The gadget is based on the von Staudt constructions also used by Mnëv [34]. 
Proof. We first build a gadget that ensures that two lines are parallel. ${ }^{12}$ Take a $C_{6}$ on vertices $p_{1}, \ldots, p_{6}$ and add edge $p_{2} p_{5}$. Let all these edges have unit length, and add two edges $p_{1} p_{3}$ and $p_{4} p_{6}$ of length 2 . In any realization of this graph, the lines through $p_{1} p_{2}$ and $p_{4} p_{5}$ are parallel, and $p_{1} / p_{5}$ and $p_{2} / p_{4}$ can get arbitrarily close together (even coincide). Now add Peaucellier linkages $L\left(p_{1}, a, p_{2}\right), L\left(p_{1}, b, p_{2}\right), L\left(p_{4}, c, p_{5}\right), L\left(p_{4}, d, p_{5}\right)$. The resulting gadget is $P(a, b, c, d)$; note that $a b$ and $c d$ are parallel in any realization of $P(a, b, c, d)$, and if we are given points $\{a, b, c, d\}$ so that $a b$ and $c d$ are parallel, and all points in $\{a, b, c, d\}$ lie within a unit disk, then $P(a, b, c, d)$ is realizable.

To build $T(a, b)$, start with vertices $a, b$, add vertices $c, u, v, w$ and edges $a u, a v, u v$ of unit length, add two Peaucellier linkages $L(a, b, u)$ and $L(a, c, v)$, and add $P(u, v, b, w)$ and $P(u, w, b, c)$. Then $|a-c|$ fulfills $|a-c| /|a-w|=$ $|a-b| /|a-u|=|a-b|$, so $|a-c|=|a-w| \cdot|a-b|=|a-b|^{2}$, since $|a-w|=|a-b|$, as $u v$ and $b w$ are parallel. Furthermore, if $a$ and $b$ have distance at most 1 , then $T(a, b)$ is realizable.

Corollary 3.8. We can build a linkage $D\left(u_{1}, u_{2}\right)$ so that $u_{1}, u_{2}$ are distinct in any realization of $D\left(u_{1}, u_{2}\right)$; moreover, for any $u_{1}, u_{2}$ which have distance at least $1 / 2^{2^{n^{2}}}$ (and distance at most 1 ), there is a realization of $D\left(u_{1}, u_{2}\right)$. The linkage $D\left(u_{1}, u_{2}\right)$ has size at most polynomial in $n$.

Proof. We take $n^{2}$ copies of $T(a, b)$ from Lemma 3.7 and chain them together by identifying $a, c$ with $a, b$ of the next device. Finally, we identify the vertices $a, b$ of the first $T(a, b)$ with the vertices $d, e$ of the Moser spindle (Figure 4) so that $a$ and $b$ have distance less than $1 / 2$ (indeed, closer to 0.47). ${ }^{13}$ Then, by the properties of $T(a, b)$, the vertices $a, c$ of the last copy of $T(a, b)$ have distance $|a-c|=|d-e|^{2^{n^{2}}}<1 / 2^{2^{n^{2}}}$.

Given vertices $u_{1}, u_{2}$ take two copies of $L$ as constructed in Lemma 3.6 on vertices $L\left(u_{1}, a, b\right)$ and $L\left(a, b, u_{2}\right)$. The resulting device $D\left(u_{1}, u_{2}\right)$ forces $u_{1}$ and $u_{2}$ to have distance at least $|d-e|^{2^{n^{2}}}>0$ and are thus distinct.

\footnotetext{
${ }^{12}$ Kempe used a parallelism gadget like this in his proof of the universality theorem that every bounded part of an algebraic curve can be traced by a suitable linkage. His parallelism gadget was flawed, however; there are many ways to repair the construction, we follow a construction due to Kapovich and Millson [25], also described in [11, Section $3.2 .2]$.

${ }^{13}$ The realization of the Moser graph is not unique; both of the diamonds can flip; to force $d$ and $e$ to be realized at distance $<1 / 2$ as shown in Figure 4 we brace the construction by adding edges $g x, x y$, and $y b$ of lengths $w(g x)=1, w(x y)=3, w(y b)=1$; this forces $g$ and $b$ to have distance at least 1, thereby forcing the intended realization of the Moser graph.
} 
Also, for any $u_{1}, u_{2}$ that have at least distance $1 / 2^{2^{n^{2}}}$, gadget $D\left(u_{1}, u_{2}\right)$ is realizable.

We are finally in a position to prove Theorem 3.1.

Proof of Theorem 3.1. It is easy to see that the problem lies in $\exists \mathbb{R}$. We reduce from STRETCHABILITY.

Suppose we are given a pseudoline arrangement $\mathcal{A}$. Create a vertex for every intersection point. For any three consecutive points $u_{1}, u_{2}, u_{3}$ along a pseudoline add the device $L\left(u_{1}, u_{2}, u_{3}\right)$. For any two intersection points $u_{1}, u_{2}$ add the device $D\left(u_{1}, u_{2}\right)$. By Lemma 3.4, we can assume that the resulting graph $G_{\mathcal{A}}$ has edges of unit length only. If $\mathcal{A}$ is stretchable, then there is a realization of $\mathcal{A}$ by straight lines in which all intersections lie within the unit disk and any two intersection points have distance at least $1 / 2^{2^{c n}}$ for some fixed $c>0$ by Lemma 1.4. But then $G_{\mathcal{A}}$ is realizable as long as $n \geq c$, because then $1 / 2^{2^{n^{2}}}<1 / 2^{2^{c n}}$. On the other hand, if we assume that $G_{\mathcal{A}}$ is realizable, then a realization contains a set of line segments whose order types correspond to $\mathcal{A}$. Since every two of these lines intersect (we included every intersection point in $G_{\mathcal{A}}$, we can extend these line segments to infinite straight lines without changing the order type, hence $\mathcal{A}$ is stretchable.

\section{$3.2 \quad$ Rigidity}

Two configurations $p, p^{\prime}: V \rightarrow \mathbb{R}^{2}$ of the same linkage $G=(V, E, w)$ are congruent if $|p(u)-p(v)|=\left|p^{\prime}(u)-p^{\prime}(v)\right|$ for all pairs $u, v \in V$. A configuration $p: V \rightarrow \mathbb{R}^{2}$ of $G=(V, E, w)$ is rigid if there is a $\varepsilon>0$ so that any configuration $p^{\prime}: V \rightarrow \mathbb{R}^{2}$ that is close to $p$ in the sense that $\left|p(v)-p^{\prime}(v)\right|<\varepsilon$ for all $v \in V$ is congruent to $p$. Informally speaking, the configuration cannot be changed by perturbing points slightly.

Abbott [1] showed that rigidity is coNP-hard using the following argument: Let ISO be the problem of deciding whether a family $f_{i}, i \in[s]$, of polynomials in $n$ variables has an isolated zero. Let $\mathrm{H}_{2} \mathrm{~N}$ be the following computational problem: given a family $f_{i}, i \in[s]$, of $s$ homogeneous polynomials in $n$ variables, is there a non-trivial zero, i.e. $\left(x_{1}, \ldots, x_{n}\right) \neq \mathbf{0}$, so that $f_{i}\left(x_{1}, \ldots, x_{n}\right)=0$ for all $i \in[s] .{ }^{14}$ Then $\mathrm{H}_{2} \mathrm{~N}$ reduces to $\overline{\mathrm{ISO}}$ based on the observation that a family of homogenous polynomials has a non-trivial zero if and only if $\mathbf{0}$ is not an isolated zero of this family [1, Corollary 5.6]. Moreover, ISO reduces to rigidity, a non-trivial reduction due to Abbott [1, Theorem 5.7], and part of his thesis on Kempe's universality theorem; the

\footnotetext{
${ }^{14}$ The name $\mathrm{H}_{2} \mathrm{~N}$ seems to be short for Hilbert's homogenous Nullstellensatz [27].
} 
reduction is in polynomial time, as long as the total degree of the polynomials is bounded by a constant. Together with Koiran's result that $\mathrm{H}_{2} \mathrm{~N}$ is NP-hard even for polynomials of total degree 2, this implies Abbott's result that rigidity is coNP-hard. However, as we are about to show, $\mathrm{H}_{2} \mathrm{~N}$ is not only NP-hard, but $\exists \mathbb{R}$-complete, so that rigidity is $\forall \mathbb{R}$-hard using the same chain of reductions that Abbott uses. The following lemma is a stepping stone to the $\exists \mathbb{R}$-hardness of $\mathrm{H}_{2} \mathrm{~N}$.

Lemma 3.9. Deciding whether a family of polynomials $f_{i}: \mathbb{R}^{n} \rightarrow \mathbb{R}, i \in[s]$ has a common root in $B^{n}(\mathbf{0}, 1)$ (the unit ball) is $\exists \mathbb{R}$-complete. We can assume that all $f_{i}$ have total degree at most 2 .

Proof. It is well-known that deciding whether a family of polynomials $g_{i}$ : $\mathbb{R}^{m} \rightarrow \mathbb{R}, i \in[s]$, has a common root is $\exists \mathbb{R}$-complete [43], even if all $g_{i}$ have total degree at most $2 .{ }^{15}$ We want to reduce this problem to the problem of deciding whether a family of polynomials $f_{i}$ has a common root in $B^{n}(\mathbf{0}, 1)$.

Suppose we are given a family of polynomials $g_{i}: \mathbb{R}^{m} \rightarrow \mathbb{R}, i \in[s]$ of total degree at most 2. By Theorem 1.3 we know that if the $g_{i}, i \in[s]$, have a common root, then such a root has distance less than $R=2^{L 2^{c n}}$ from the origin, where $L$ is an upper bound on the bit-lengths of the coefficients of the $g_{i}$ and $c>0$ is some fixed constant (we use that the $g_{i}$ have total degree $2)$. Let $t=\lceil n \log c+\log L\rceil+1\left(\right.$ so $\left.2^{2^{t}}>R^{2}\right)$ and define

$$
f_{i}\left(x_{1}, \ldots, x_{m}, y_{0}, y_{1}, \ldots, y_{t}\right):=y_{0}^{2} g_{i}\left(x_{1} / y_{0}, \ldots, x_{m} / y_{0}\right)
$$

for $i \in[s]$ and

$$
f_{s+1}\left(x_{1}, \ldots, x_{m}, y_{0}, y_{1}, \ldots, y_{t}\right):=y_{t}-1 / 2
$$

and

$$
f_{s+1+i}\left(x_{1}, \ldots, x_{m}, y_{0}, y_{1}, \ldots, y_{t}\right):=y_{i-1}-y_{i}^{2}
$$

for $i \in[t]$. Note that all the $f_{i}$ have total degree at most 2 .

If the $f_{i}, i \in[s+1+t]$ have a common root $\left(x_{1}, \ldots, x_{m}, y_{0}, y_{1}, \ldots, y_{t}\right)$, then $y_{t}=1 / 2$, since $f_{s+1}=0$, and $y_{t-i}=2^{-2^{i}}$ for $i \in[t]$ since $f_{s+i+1}=0$ for $i \in[t]$. In particular, $y_{0}>0$, so $f_{i}=0$ implies that $g_{i}\left(x_{1} / y_{0}, \ldots, x_{m} / y_{0}\right)=0$, for all $i \in[s]$, so $\left(x_{1} / y_{0}, \ldots, x_{m} / y_{0}\right)$ is a common root of the $g_{i}, i \in[s]$.

On the other hand, assume that the $g_{i}$ have a common root $\left(x_{1}^{\prime}, \ldots, x_{m}^{\prime}\right)$, we can assume that $\left(x_{1}^{\prime}, \ldots, x_{m}^{\prime}\right)$ has distance less than $R$ from the origin. Let $y_{i}:=2^{-2^{t-i}}$ for $i \in\{0\} \cup[t]$ and $x_{i}:=x_{i}^{\prime} y_{0}$. By definition, all $f_{i}=0$,

\footnotetext{
${ }^{15}$ This is a folklore result; for example, it is easy to see that STRETCHABILITY can be rephrased like this. The version in the Blum-Shub-Smale model can be found in [6].
} 
$i \in[s+1+t]$. We only need to verify that $\left(x_{1}, \ldots, x_{m}, y_{0}, y_{1}, \ldots, y_{t}\right) \in$ $B^{m+1+t}(\mathbf{0}, 1)$. Now $\sum_{i=0}^{t} y_{i}^{2} \leq \sum_{i=0}^{\infty} 4^{-2^{i}}=1 / 4+1 / 16+1 / 256+1 / 65536+$ $\ldots \leq 1 / 4+1 / 8=5 / 8$. Also, $\sum_{i=1}^{m} x_{i}^{2}=y_{0}^{2} \sum_{i=1}^{m} x_{i}^{\prime 2} \leq y_{0}^{2} R^{2} \leq\left(1 / R^{2}\right)^{2} R^{2}=$ $1 / R^{2}$. So $\left(x_{1}, \ldots, x_{m}, y_{0}, y_{1}, \ldots, y_{t}\right)$ has distance at most $\sqrt{5 / 8+1 / R^{2}}<1$ from the origin (assuming that $R \geq 2$ ), and we have found a common root of the $f_{i}$ in $B^{m+1+t}(\mathbf{0}, 1)$.

Corollary 3.10. $\mathrm{H}_{2} \mathrm{~N}$ is $\exists \mathbb{R}$-complete.

Proof. It is easy to see that $\mathrm{H}_{2} \mathrm{~N}$ belongs to $\exists \mathbb{R}$, so we only have to show that it is $\exists \mathbb{R}$-hard. By Lemma 3.9 deciding whether a family of polynomials $f_{i}: \mathbb{R}^{n} \rightarrow \mathbb{R}, i \in[s]$, has a common root $\mathbf{x} \in B^{n}(\mathbf{0}, 1)$ is $\exists \mathbb{R}$-complete, even if all $f_{i}$ have total degree at most 2 .

Using a standard transformation, we can turn each $f_{i}$ into a homogenous polynomial, just adding one additional variable $y_{0}$ : define

$$
g_{i}\left(x_{1}, \ldots, x_{n}, y_{0}\right):=y_{0}^{4} f_{i}\left(x_{1} / y_{0}, \ldots, x_{n} / y_{0}\right),
$$

$i \in[s]$. Then $\left(g_{i}\right)_{i \in[s]}$ is a family of homogeneous polynomials of total degree 4 and $\left(x_{1}, \ldots, x_{n}, y_{0}\right)$ is a common root of all $g_{i}, i \in[s]$, if and only if either $y_{0}=0$ or $y_{0} \neq 0$ and $\left(x_{1} / y_{0}, \ldots, x_{n} / y_{0}\right)$ is a common root of the $f_{i}, i \in[s]$. This does not yet meet our goal, since we can have non-trivial common roots of the $g_{i}$ that do not correspond to any common roots of the $f_{i}$ : just let $y_{0}=0$ and $\left(x_{1}, \ldots, x_{n}\right) \neq \mathbf{0}$. This can be remedied by making $y_{0}$ an upper bound of the $x_{i}$; let

$$
g_{s+1}\left(x_{0}, x_{1}, \ldots, x_{n}, y_{0}\right)=y_{0}^{4}-x_{0}^{4}-\left(\sum_{i=1}^{n} x_{i}^{2}\right)^{2}
$$

adding a new variable $x_{0}$.

Let $\left(x_{0}, x_{1}, \ldots, x_{n}, y_{0}\right)$ be a non-trivial common root of the $g_{i}, i \in[s+1]$ (adding $x_{0}$ to the list of variables of the $g_{i}$ with $i \in[s]$ ). If $y_{0}=0$, then $x_{0}=x_{1}=\cdots x_{n}=0$ (because $g_{s+1}=0$ ), so $\left(x_{0}, x_{1}, \ldots, x_{n}, y_{0}\right)=\mathbf{0}$ and the root is trivial. Hence, we must have $y_{0} \neq 0$. But then $g_{i}\left(x_{1}, \ldots, x_{n}, y_{0}\right)=0$ implies that $f_{i}\left(x_{1} / y_{0}, \ldots, x_{n} / y_{0}\right)=0$, so $\left(x_{1} / y_{0}, \ldots, x_{n} / y_{0}\right)$ is a common root of the $f_{i}$. Moreover, since $g_{s+1}=0$, we have that $y_{0}^{4}>\left(\sum_{i=1}^{n} x_{i}^{2}\right)^{2}$, so $\sum_{i=1}^{n}\left(x_{i} / y_{0}\right)^{2}<1$ which implies that $\left(x_{1} / y_{0}, \ldots, x_{n} / y_{0}\right) \in B^{n}(\mathbf{0}, 1)$.

For the reverse direction, assume that we are given a root $\left(x_{1}, \ldots, x_{n}\right) \in$ $B^{n}(\mathbf{0}, 1)$ of the $f_{i}, i \in[s]$. Let $y_{0}:=1$ and $x_{0}:=\left(y_{0}^{4}-\left(\sum_{i=1}^{n} x_{i}^{2}\right)^{2}\right)^{1 / 4}$ (which is defined, since $\left.\sum_{i=1}^{n} x_{i}^{2}<1\right)$. By definition, $g_{s+1}\left(x_{0}, x_{1}, \ldots, x_{n}, y_{0}\right)=0$ and $g_{i}\left(x_{0}, x_{1}, \ldots, x_{n}, y_{0}\right)=0$. So $\left(x_{0}, x_{1}, \ldots, x_{n}, y_{0}\right)$ is a non-trivial common root of the $g_{i}$ which is what we had to prove. 
Thus following Abbott's construction [1, Theorem 5.7 $]^{16}$, we get $\forall \mathbb{R}$ hardness of rigidity.

Theorem 3.11. Rigidity in $\mathbb{R}^{2}$ is $\forall \mathbb{R}$-complete.

To complete the proof of Theorem 3.11 it remains to show that rigidity lies in $\forall \mathbb{R}$. The formal definition of rigidity given at the beginning of this section gives us an $\exists \forall$ formula for rigidity. We use a lemma that allows us to convert the leading existential quantifier into a group of universal quantifiers in this case.

Lemma 3.12 (Schaefer [41]). Suppose

$$
\Phi\left(\varepsilon, y_{1}, \ldots, y_{\ell}\right)=\left(\forall x_{1}, \ldots, x_{k}\right) \varphi\left(\varepsilon, x_{1}, \ldots, x_{k}, y_{1}, \ldots, y_{\ell}\right),
$$

is such that $\Phi\left(\varepsilon, y_{1}, \ldots, y_{\ell}\right)$ implies $\Phi\left(\varepsilon^{\prime}, y_{1}, \ldots, y_{\ell}\right)$ for all $\varepsilon>\varepsilon^{\prime}>0$. Then we can find a quantifier-free formula $\psi\left(\varepsilon, x_{1}, \ldots, x_{k}, y_{1}, \ldots, y_{\ell}, z_{1}, \ldots, z_{m}\right)$ of length at most $|\varphi|+d m$ in time $|\varphi|+d m$, where $m=c n^{3} \log |\varphi|$ so that

$$
(\exists \varepsilon>0)\left(\forall x_{1}, \ldots, x_{k}\right) \varphi\left(\varepsilon, x_{1}, \ldots, x_{k}, y_{1}, \ldots, y_{\ell}\right)
$$

is equivalent to

$$
\left(\forall z_{1}, \ldots, z_{m}\right)(\forall \varepsilon)\left(\forall x_{1}, \ldots, x_{k}\right) \psi\left(\varepsilon, x_{1}, \ldots, x_{k}, y_{1}, \ldots, y_{\ell}, z_{1}, \ldots, z_{m}\right)
$$

for some fixed constants $c, d>0$.

Remark 3.13. Lemma 3.12 is a consequence of the lemma by Grigor'ev and Vorobjov stated here as Theorem 1.3.

Proof of Theorem 3.11. We already showed $\forall \mathbb{R}$-hardness, based on the reduction by Abbott [1, Theorem 5.7]. We still have to show that the problem belongs to $\forall \mathbb{R}$. The input is a particular configuration of some linkage $G$ given as a position $p(v)$ assigned to each vertex $V(G)=\left\{v_{1}, \ldots, v_{n}\right\}$. As a computational problem, the input needs to be finite, so we assume that the coordinates of $p(v)$ are algebraic real numbers. The configuration is rigid if there is an $\varepsilon>0$ so that for all $p^{\prime}(v), v \in V(G)$ with

(i) $\left|p^{\prime}(v)-p(v)\right| \leq \varepsilon$ for all $v \in V$ and

(ii) $\left|p^{\prime}(u)-p^{\prime}(v)\right|=|p(u)-p(v)|$ for all $u v \in E(G)$,

\footnotetext{
${ }^{16}$ The reduction is polynomial time, since our polynomials have total degree 2 .
} 
we have that the configurations $p^{\prime}$ and $p$ are congruent. This characterization is not $\forall \mathbb{R}$ because of the leading existential quantifier. However, it is monotone in $\varepsilon$ in the sense of Lemma 3.12 so we can convert the existential quantifier into a block of universal quantifiers of length polynomial in $n$ and the original formula. This if sufficient to show that the problem lies in $\forall \mathbb{R}$.

Remark 3.14. The proof of Theorem 3.11 can probably be strengthened to show that recognizing the rigidity of unit distance graphs is $\forall \mathbb{R}$-complete. However, the construction will be a rather lengthy, technical recreation of Kempe's proof with unit distance graphs, so the author decided to leave this for a different occasion.

\section{Open Questions}

Approximation Saxe introduced an approximate version of realizability for linkages, but we can apply it to both graph and linkage realizability: call a graph or linkage $\varepsilon$-approximately realizable if the vertices can be placed so that for every edge, $1-\varepsilon<\operatorname{len}(e) / \ell(e)<1+\varepsilon$, where len $(e)$ is the actual length of edge $e$ in the straight-line drawing and $\ell(e)$ is the intended length. For unit distance graphs, this leads to the notion of a $\varepsilon$-unit distance graph.

Question 4.1. Is recognizing $\varepsilon$-approximately realizable graphs or linkages still $\exists \mathbb{R}$-hard? Is recognizing $\varepsilon$-unit distance graphs $\exists \mathbb{R}$-hard?

The case $\varepsilon=1 / 2^{2^{O\left(n^{c}\right)}}$ would be a good starting point in view of Lemma 1.4. Saxe [40] showed that $1 / 9$-approximate realizability of linkages in $\mathbb{R}$ is NP-complete (this proof requires a different encoding from the one outlined in Remark 3.2, since the partition problem can be approximated well).

Higher Dimensions All of our results were proved for $\mathbb{R}^{2}$; since $\mathbb{R}$ is wellunderstood, this leaves the question of how hard it is to decide problems on linkages and graphs in higher-dimensional spaces.

Acknowledgements I would like to thank the anonymous referee for pointing out the universality papers by Jordan and Steiner [23] and Kapovich and Millson [25]. 


\section{References}

[1] Timothy Good Abbott. Generalizations of Kempe's universality theorem. Master's thesis, Massachusetts Institute of Technology, Dept. of Electrical Engineering and Computer Science, 2008.

[2] Saugata Basu, Richard Pollack, and Marie-Françoise Roy. Algorithms in real algebraic geometry, volume 10 of Algorithms and Computation in Mathematics. Springer-Verlag, Berlin, second edition, 2006.

[3] Daniel Bienstock. Some provably hard crossing number problems. Discrete Comput. Geom., 6(5):443-459, 1991.

[4] Anders Björner, Michel Las Vergnas, Bernd Sturmfels, Neil White, and Günter M. Ziegler. Oriented matroids, volume 46 of Encyclopedia of Mathematics and its Applications. Cambridge University Press, Cambridge, second edition, 1999.

[5] Johannes Blömer. A probabilistic zero-test for expressions involving roots of rational numbers. In Algorithms-ESA '98 (Venice), volume 1461 of Lecture Notes in Comput. Sci., pages 151-162. Springer, Berlin, 1998.

[6] Lenore Blum, Mike Shub, and Steve Smale. On a theory of computation and complexity over the real numbers: NP-completeness, recursive functions and universal machines. Bull. Amer. Math. Soc. (N.S.), 21(1):1-46, 1989.

[7] Sergio Cabello, Erik D. Demaine, and Günter Rote. Planar embeddings of graphs with specified edge lengths. J. Graph Algorithms Appl., 11(1):259-276 (electronic), 2007.

[8] John Canny. Some algebraic and geometric computations in pspace. In STOC '88: Proceedings of the twentieth annual ACM symposium on Theory of computing, pages 460-469, New York, NY, USA, 1988. ACM.

[9] V. Chvátal, D. A. Klarner, and D. E. Knuth. Selected combinatorial research problems. Technical Report STAN-CS-72-292, Computer Science Department, Stanford University, 1972.

[10] Robert Connelly and Erik D. Demaine. Geometry and topology of polygonal linkages. In Jacob E. Goodman and Joseph O'Rourke, editors, Handbook of Discrete and Computational Geometry. CRC Press, 2004. 
[11] Erik D. Demaine and Joseph O'Rourke. Geometric folding algorithms. Cambridge University Press, Cambridge, 2007. Linkages, origami, polyhedra.

[12] Giuseppe Di Battista and Luca Vismara. Angles of planar triangular graphs. SIAM Journal on Discrete Mathematics, 9(3):349-359, August 1996.

[13] Peter Eades and Nicholas C. Wormald. Fixed edge-length graph drawing is NP-hard. Discrete Appl. Math., 28(2):111-134, 1990.

[14] David Eppstein. Unit distance graphs, 2010. Blog posting at http:// 11011110.livejournal.com/188807.html (accessed September 10, 2010).

[15] P. Erdős and M. Simonovits. On the chromatic number of geometric graphs. Ars Combin., 9:229-246, 1980.

[16] Paul Erdős, Frank Harary, and William T. Tutte. On the dimension of a graph. Mathematika, 12:118-122, 1965.

[17] Xiaoshan Gao and Changcai Zhu. Automated generation of Kempe linkage and its complexity. J. Comput. Sci. Tech., 14(5):460-467, 1999.

[18] Jacob E. Goodman and Joseph O'Rourke, editors. Handbook of discrete and computational geometry. Discrete Mathematics and its Applications (Boca Raton). Chapman \& Hall/CRC, Boca Raton, FL, second edition, 2004.

[19] Jacob E. Goodman, Richard Pollack, and Bernd Sturmfels. Coordinate representation of order types requires exponential storage. In STOC '89: Proceedings of the twenty-first annual ACM symposium on Theory of computing, pages 405-410, New York, NY, USA, 1989. ACM.

[20] D. Yu. Grigor'ev and N. N. Vorobjov. Solving systems of polynomial inequalities in subexponential time. J. Symb. Comput., 5(1-2):37-64, 1988.

[21] S. L. Greitzer H. S. M. Coxeter. Geometry Revisited. The Mathematical Association of America, Washington, D.C., 1967.

[22] Hoon Hong. Comparison of several decision algorithms for the existential theory of the reals. Technical Report 91-41, RISC-Linz, Johannes Kepler University, Linz, Austria, 1991. 
[23] D. Jordan and M. Steiner. Configuration spaces of mechanical linkages. Discrete Comput. Geom., 22(2):297-315, 1999.

[24] Matthew Kahle. Algorithm for embedding a graph with metric constraints, 2010. Posting on mathoverflow at http://mathoverflow.net/questions/33043 (accessed September 9,2010$)$.

[25] Michael Kapovich and John J. Millson. Universality theorems for configuration spaces of planar linkages. Topology, 41(6):1051-1107, 2002.

[26] Alfred Bray Kempe. How to Draw a Straight Line. Classics in Mathematics Education. MacMillan, London, 1977.

[27] Pascal Koiran. The complexity of local dimensions for constructible sets. J. Complexity, 16(1):311-323, 2000. Real computation and complexity (Schloss Dagstuhl, 1998).

[28] Monique Laurent. A tour d'horizon on positive semidefinite and Euclidean distance matrix completion problems. In Topics in semidefinite and interior-point methods (Toronto, ON, 1996), volume 18 of Fields Inst. Commun., pages 51-76. Amer. Math. Soc., Providence, RI, 1998.

[29] Paul Lemke, Steven S. Skiena, and Warren D. Smith. Reconstructing sets from interpoint distances. In Discrete and computational geometry, volume 25 of Algorithms Combin., pages 507-631. Springer, Berlin, 2003.

[30] David Little. Peaucellier's linkage, 2005. Applet at http://www . math . psu.edu/dlittle/java/parametricequations/linkage.html.

[31] Hiroshi Maehara. Note on induced subgraphs of the unit distance graph $E^{n}$. Discrete Comput. Geom., 4(1):15-18, 1989.

[32] C.J.H. McDiarmid and T. Müller. The number of bits needed to represent a unit disk graph, 2010.

[33] Bhubaneswar Mishra. Computational real algebraic geometry. In Handbook of discrete and computational geometry, CRC Press Ser. Discrete Math. Appl., pages 537-556. CRC, Boca Raton, FL, 1997.

[34] N. E. Mnëv. The universality theorems on the classification problem of configuration varieties and convex polytopes varieties. In Topology and geometry-Rohlin Seminar, volume 1346 of Lecture Notes in Math., pages 527-543. Springer, Berlin, 1988. 
[35] Christos H. Papadimitriou. Computational complexity. Addison-Wesley Publishing Company, Reading, MA, 1994.

[36] James Renegar. On the computational complexity and geometry of the first-order theory of the reals. I. Introduction. Preliminaries. The geometry of semi-algebraic sets. The decision problem for the existential theory of the reals. J. Symbolic Comput., 13(3):255-299, 1992.

[37] James Renegar. On the computational complexity and geometry of the first-order theory of the reals. II. The general decision problem. Preliminaries for quantifier elimination. J. Symbolic Comput., 13(3):301-327, 1992.

[38] James Renegar. On the computational complexity and geometry of the first-order theory of the reals. III. Quantifier elimination. J. Symbolic Comput., 13(3):329-352, 1992.

[39] Jürgen Richter-Gebert. Mnëv's universality theorem revisited. Sém. Lothar. Combin., 34, 1995.

[40] James B. Saxe. Embeddability of weighted graphs in $k$-space is strongly NP-hard. In Seventeenth Annual Allerton Conference on Communication, Control, and Computing, Proceedings of the Conference held in Monticello, Ill., October 10-12, 1979, pages xiv+1036, Urbana, 1979. University of Illinois Department of Electrical Engineering. Proceedings of the International School of Physics "Enrico Fermi", LXX*.

[41] Marcus Schaefer. The real logic of drawing graphs. Unpublished Manuscript.

[42] Marcus Schaefer. Complexity of some geometric and topological problems. In David Eppstein and Emden R. Gansner, editors, Graph Drawing, volume 5849 of Lecture Notes in Computer Science, pages 334-344. Springer, 2009.

[43] Marcus Schaefer and Daniel Štefankovič. Fixed points, Nash equilibria, and the existential theory of the reals. Unpublished manuscript.

[44] Peter W. Shor. Stretchability of pseudolines is NP-hard. In Applied geometry and discrete mathematics, volume 4 of DIMACS Ser. Discrete Math. Theoret. Comput. Sci., pages 531-554. Amer. Math. Soc., Providence, RI, 1991. 
[45] Michael Sipser. Introduction to the Theory of Computation. Course Technology, 2nd edition, 2005.

[46] Alexander Soifer. The mathematical coloring book. Springer, New York, 2009. Mathematics of coloring and the colorful life of its creators, With forewords by Branko Grünbaum, Peter D. Johnson, Jr. and Cecil Rousseau.

[47] J. J. Sylvester. On recent discoveries in mechanical conversion of motion. In Proceedings of the Royal Institution of Great Britain, pages 179-198, London, 1875. Talk delivered January 23rd, 1874.

[48] László A. Székely. A successful concept for measuring non-planarity of graphs: the crossing number. Discrete Math., 276(1-3):331-352, 2004.

[49] Sue Whitesides. Algorithmic issues in the geometry of planar linkage movement. Australian Computer Journal, 24(2):42-50, 1992.

[50] Yechiam Yemini. Some theoretical aspects of position-location problems. In 20th Annual Symposium on Foundations of Computer Science (San Juan, Puerto Rico, 1979), pages 1-8. IEEE, New York, 1979. 